\title{
Parametrically Generating New Instances \\ of Traditional Chinese Private Gardens that Replicate Selected Socio-Spatial and Aesthetic Properties
}

\author{
Rongrong Yu $\mathbf{u}^{1} \cdot$ Michael J. Ostwald ${ }^{1}$. \\ Ning Gu ${ }^{1}$
}

Published online: 1 August 2015

(C) Kim Williams Books, Turin 2015

\begin{abstract}
This paper describes the use of a parametric system for generating garden plans that replicate selected socio-spatial characteristics and aesthetic properties of traditional Chinese private gardens (TCPGs). To achieve this, the spatial characteristics of three historic TCPGs are first mathematically derived using connectivity analysis, a variation of a space syntax technique. The data developed through this process is then used to shape the rules of a parametric system to generate new garden plans with similar spatial connectivity values and structures. While these new plans capture some of the socio-spatial features of the TCPG, the other important characteristic of these gardens is a particular level of visual complexity. Using fractal analysis, the characteristic visual complexity of the newly generated garden plans is then compared with the historic cases, to assess the success of the system in aesthetic terms. Through this three-stage process (syntactical derivation, parametric generation and fractal analysis) the paper demonstrates a method for capturing selected spatial and aesthetic properties in a parametric system and also provides new tools for landscape design in the context of specific historical sites and approaches.
\end{abstract}

Keywords Traditional Chinese private gardens $\cdot$ Space syntax · Connectivity analysis · Parametric generation · Fractal analysis

Rongrong $\mathrm{Yu}$

rongrong.yu@uon.edu.au

Michael J. Ostwald

michael.ostwald@newcastle.edu.au

Ning Gu

ning.gu@newcastle.edu.au

1 School of Architecture and Built Environment, The University of Newcastle, University Drive,

Callaghan, NSW 2308, Australia

Birkhäuser 


\section{Introduction}

In landscape architecture, one of the most famous and admired historic garden types is the traditional Chinese private garden (TCPG) (Rinaldi 2011; Hunt 2012). The origin of the TCPG is typically traced to the Tang Dynasty (circa 800 AD) although examples of private hunting reserves, with artificially constructed landscapes, can be found in China in the eleventh century BC. By the seventeenth century AD, the TCPG had become a special type of landscaped space, characterised in part by its rich spatial arrangement and its associated aesthetic properties. A typical TCPG is made up of a dense network of paths and spaces, punctuated with artificial landscape features, ponds and small streams, paved squares and covered bridges, all of which are organised in a relatively small and clearly defined area (Fig. 1). The TCPG is today renowned for exhibiting high levels of spatial complexity and variety, properties that have led to them being accepted as having unique experiential qualities (Peng 1986; Tong 1997). Furthermore, such is their importance that many recent landscape architecture texts describe how the features of the TCPG might be mimicked or developed in more contemporary settings (Feng 2010; Chen 2011; Sui and Xun 2011).

In parallel with this growth in interest from designers, researchers have analysed the TCPG from various qualitative perspectives (Tong 1997; Hunt 2012) and a much smaller number of studies have also examined their spatial properties using quantitative methodologies (Chang 2006; Lu 2009, 2010; Li 2011; Wang and Wang 2013). For example, Keswick (1978) and Zhou (1999) separately explored the spatial character of TCPGs from a historical and social perspective. In the latter case, with regard to the quantitative methods, multiple attempts have been made to understand the dense spatial configurations in TCPGs and the variable changing vistas experienced while navigating through them (Peng 1986; Li 2011; Chen 2012). Syntactical techniques have also been employed to examine specific spatial qualities in TCPGs (Chang 2006; Chen 2012; Wang and Wang 2013) and to support grammatical interpretations of their formal compositions (Lu 2009, 2010) or visual perceptual characters (Li 2011).
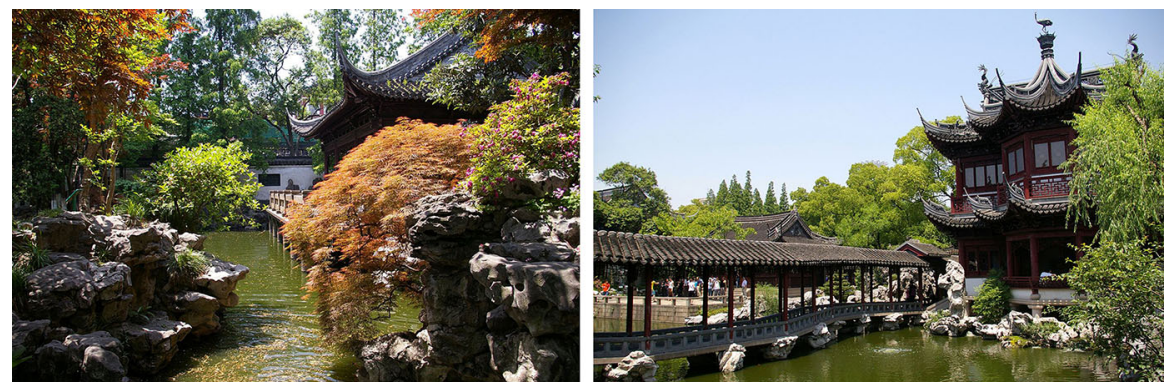

Fig. 1 Yuyuan Gardens in Shanghai. Source: Photographs by Jakub Halon, licensed under Creative Commons 
Despite this growing body of research, the spatial qualities that make TCPGs unique have rarely been measured and generalised mathematically. Without such a set of measures, their spatial and aesthetic properties cannot be replicated in current landscape design practice or maintained as part of the restoration process for these sensitive heritage structures. Thus, the purpose of the present paper is to begin to develop a system that can generate new TCPG plans, using parametric design, which capture selected aspects of the spatial structure and visual character of the originals.

The starting point for the present research is a set of three sixteenth-century TCPGs in southern China: Yuyuan (Shanghai) (Fig. 1), Zhuozhengyuan (Suzhou) and Liuyuan (Suzhou). These cases are all well-known examples, with two of them being regarded as amongst the most famous of their type. The selected TCPG cases are all typical examples of Ming and Qing dynasty private gardens in southern China (Fu et al. 2002). These three are not only from the same broad geographic region and era, but all three have total areas of over $20,000 \mathrm{~m}^{2}$. This last feature is important because it helps to ensure that there is a sufficient volume of data to produce statistically valid results using the methods applied in this paper. The three historic TCPGs are used in the first stage of this research to derive typical spatial properties, then in the second as a generative foundation and rule set, and finally, in the third stage, as the basis for comparative aesthetic testing.

The paper is structured in three stages which replicate the steps in the research method. The first stage, syntactical derivation, uses connectivity graphs to measure three properties of each historic TCPG. As part of the process, different garden spaces are categorised into various spatial-types-effectively different kinds of areas in the landscape. Then an inequality genotype is developed for each TCPG and the mathematical value ranges of the measurements for each spatial type are identified. These values are used in the second stage, parametric generation, where rules are developed to create nine new designs which feature the same spatial structures identified in the historic TCPGs. In the final stage of the research, the new designs and the three originals are all measured using the box-counting method to determine their fractal dimensions. This allows for the characteristic or typical spread of visual information in each to be compared, providing a mathematical assessment of the degree to which the new cases visually resemble the historic ones. Each of the three methods used in the paper-connectivity graphs, parametric rules and the box-counting method-are presented at the start of their respective stages in the paper. The three historic TCPGs are introduced in the first stage of the research.

The ultimate purpose of the three-stage process described in the present paper is to begin to develop and test a method for generating new designs that conform to the styles and spatio-structural qualities of TCPGs. As only a limited set of spatial parameters are derived from the TCPG for this research, and as other factors are known to contribute to their elaborate plan forms, the final result will only be indicative of the potential of the method and of its relative success. Furthermore, there are several practical limitations to the research that must be acknowledged. First, this research is solely concerned with the plans of TCPGs, and the syntactical, generative and analytical techniques used are ones that are ideal for working with two-dimensional representations of space. Second, the syntactical properties 
considered are limited to just three measures associated with connectivity between types of spaces. Thus, the full range of social interpretations that could otherwise be developed using this method are not possible in this case (Hillier and Hanson 1984; Ostwald 2011). In addition, as the focus of the analysis is on spatial types; it is not concerned with the location or distribution of specific landscape elements (statues, ponds, streams etc.) that are outside the network of spatial relations present in the accessible sections of a TCPG. The third issue that should be noted is that while fractal analysis is used in the paper to test the parametrically generated plans, it does not shape the parameters. Thus, the aesthetic properties of the new plans are a result of spatial connectivity and type-related characteristics, and the third stage measures whether this, in itself, produces an outcome that is visually similar to the originals. Finally, a sample of only three historic TCPGs is used to generate the initial values used for this study. A larger sample would provide a stronger statistical basis for the stage 1 comparison and it could also be used to develop a more nuanced set of parameters that would take into account further factors. Nevertheless, as the paper demonstrates, three cases are sufficient to uncover a strong and generalizable pattern of results that can be used to develop new TCPGs which conform to selected social and aesthetic properties of the originals.

\section{Stage 1: Syntactical Derivation}

Space syntax is a theory and associated set of techniques that developed unique architectural and urban applications of graph mathematics for the analysis of the social structure of space (Hillier and Hanson 1984; Hillier 1995). Extensively refined over the last few decades, space syntax methods have been widely applied in research in urban planning, architectural design and landscape design, amongst other areas. One of the key characteristics of the space syntax approach is that it provides a way of understanding architectural and urban spatial configurations by translating their properties into topological graphs which can then be mathematically analysed and socially interpreted (Hanson 1998).

One of the earliest syntactical analytical techniques, known as a Convex Graph or a Justified Plan Graph, creates a graph from a set of nodes, which represent spaces, and the connections between them, representing a type of boundary condition which might be trafficable, permeable or visual depending on the chosen application. There are multiple variations of this technique, including those pertaining to visually-defined spaces (convex spaces), functionally defined rooms, or functional zones in a plan or a landscape (Hanson 1998; Bafna 2003; Minor and Urban 2007). In all cases, once the chosen spaces are represented by a graph, then various mathematical properties of the graph may be derived from it (Hillier and Hanson 1984; Hillier and Kali 2006; Ostwald 2011). For example, the step depth of each node in the graph can be determined, then the total depth $(T D)$, mean depth $(M D)$ and integration $(i)$ values calculated for each node. These values reflect the relative role of each space within the larger structure of the plan. For example, the step depth suggests the connectivity distance from the entrance node, the mean depth is the average depth for each node which represents the degree of isolation of the spaces. 
While the degree of integration is suitable for comparing each space with the other parts in a distributed plan and can be used to develop an inequality genotype, which is a hierarchical determination of the structure of the spaces in a graph (Bafna 2001).

While space syntax techniques have typically been formulated to analyse spaces that have underlying functional properties associated with efficiency or control (of movement or access), the TCPG is not structured in this way. Arguably, the purpose of the TCPG is to provide a space for informal wandering and contemplation. The design of the TCPG emphasises the joy of an uncertain spatial experience, with paths snaking between areas in the garden seemingly serving to evoke a sense of delight or mystery (Keswick et al. 2003). To do this, the TCPG features several recurring design elements and strategies that collectively constitute a loose spatial pattern. For example, the pavilions and covered spaces in the TCPG are often strategically placed around bodies of water, which are themselves sited at the centre of the garden. Within the trafficable parts of the gardens, large courtyards are commonly surrounded by smaller spaces (Guo 2014). Covered or semi-enclosed structures punctuate the networks of paths between these courtyards, including pavilions lined with lattice windows and covered narrow corridors (Sun 2012). These spatial configurations and relations, which are repeated in variations in many TCPGs, provide the unique experiential quality observed by people when moving through the gardens (Keswick et al. 2003). They also evoke the sense of a linear navigation path, which is sequenced or organized to create a changing rhythm that shifts from small, tight spaces, to larger, open ones and then back again (Guo 2014; Chen 2012). Because of these properties of the TCPG, for the present paper a variation of the standard connectivity graph technique for analysing functional areas was selected. However, because TCPGs do not possess distinct functional zoneseffectively all spaces serve as passive recreation or contemplation areas-their areas were differentiated in terms of spatial types in accordance with conventional reading of the features (Keswick et al. 2003; Guo 2014).

For the present research, each of the three historic TCPGs were analysed and a set of six recurring spatial types identified: (1) large rooms, (2) small rooms, (3) pavilions, (4) yards and squares, (5) covered corridors and (6) pathways. Large and small "Rooms" are physically and often visually defined in plan by walls and landscape elements. In elements, in the three TCPGs studied, there were two clearly distinguishable types to these rooms, hence the "large" and "small" descriptors. However, the definition of a large or small room is not solely dependent on its area. Both area and inclusions or constituent parts are considered as part of the classification process. Thus, the large room is indeed typically bigger in area than the small, but importantly, it also usually features a courtyard with some landscape elements in it. Therefore large rooms are those which provide a relatively complete and independent living space, whereas small rooms, even if they are close to the area of their bigger counterparts, do not have these features. The "pavilion" spatial type includes covered or roofed structures (typically without extensive walls) which dominate a defined "room" in the garden. "Yards and squares" are open, paved spaces which are less clearly defined, and are smaller in scale than the garden "rooms". The final two types are variations of long, narrow spaces, the first of which is a roofed or "covered corridor" and the second is an uncovered, often 
meandering "pathway". The extent of these corridor types is defined by the surface treatment of the ground plane (typically paving stones) and low landscape walls and planting.

Amongst the six spatial types, the pathway is the most complex to define because many of the paths in TCPGs are very short, effectively only providing an intermediate link between two other major elements. Thus, rather than add many hundreds of separate paths to each garden structure, paths were only counted if they were necessary for connecting two spaces that would not otherwise have any connections to the graph. For example, in Fig. 3, path 76 is counted as an independent space because it is the only connection between spaces 20 and 22 . Conversely, the short path south of space 37 is not counted because it is simply the junction between space 37, and paths 87 and 106 .

With these six spatial types as a basis, plan analysis of the three historic TCPGs was undertaken using UCL Depthmap software, the conventional space syntax method. An example of the process, as undertaken for the Yuyuan garden, is depicted in Figs. 2, 3, 4 and 5. In Fig. 5 the exterior is presented as the carrier level in the graph; and the principal for calculating the total depth $(T D)$ of a space requires that, if it can fit into different depth levels, the priority is given to the lowest level. The Mean results were derived for each measure for each spatial type and the standard deviation recorded (Tables 1, 2, 3). Each of the three cases and sets of results are described in more detail hereafter in what follows.

Fig. 2 Yuyuan Garden plan, Shanghai

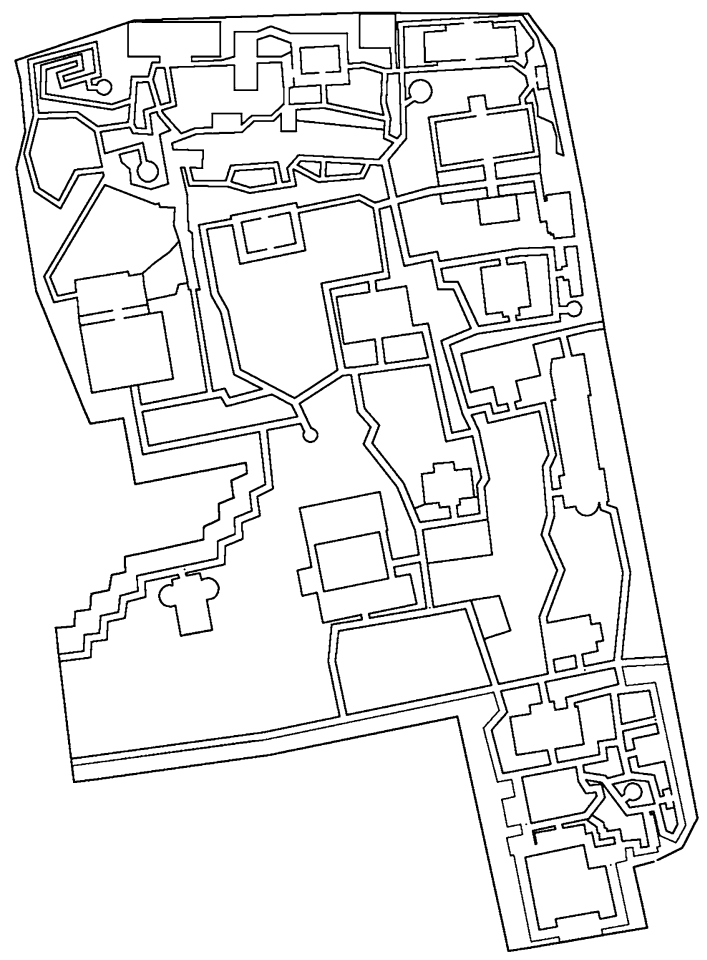




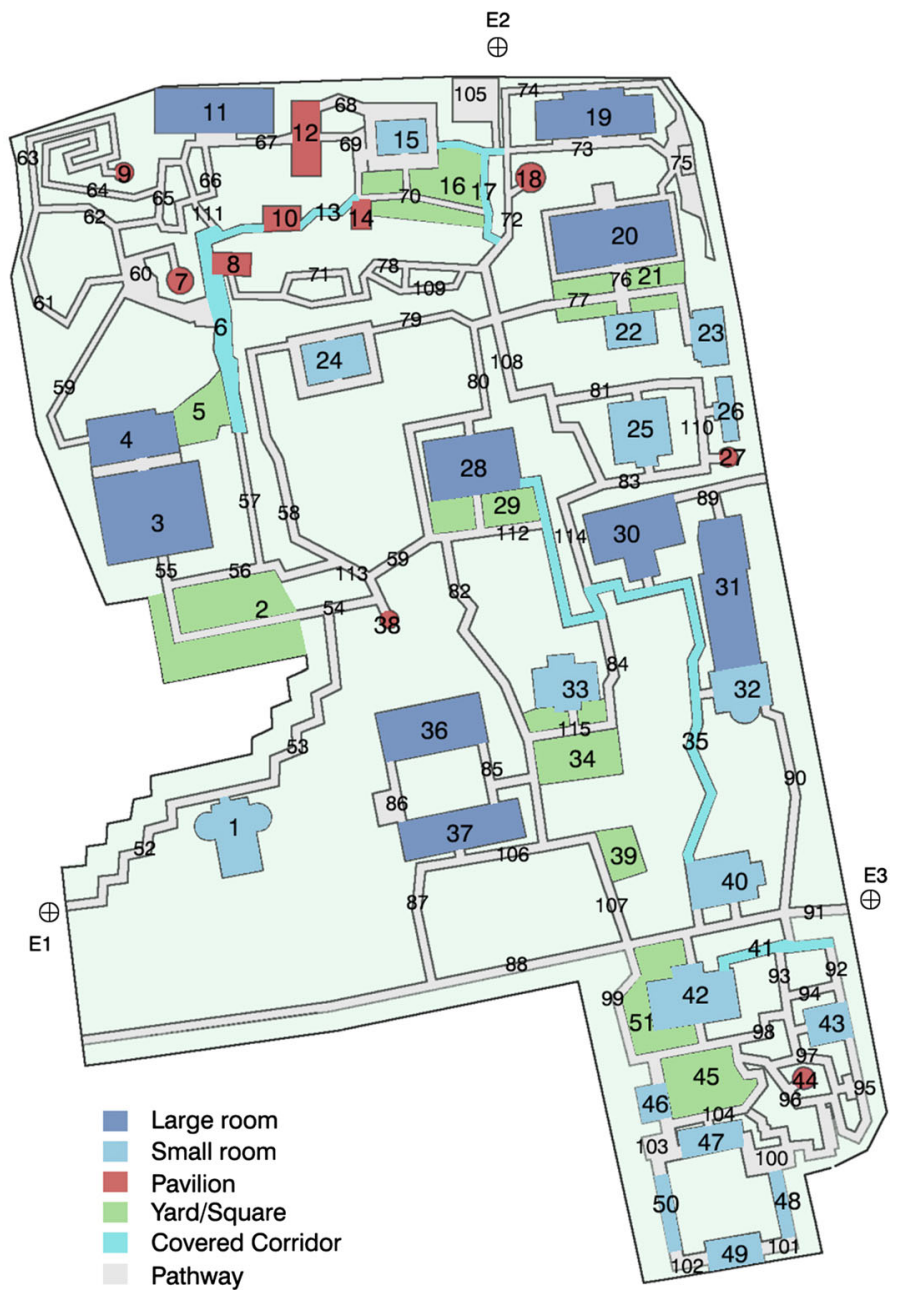

Fig. 3 Yuyuan Garden plan, colour coded and annotated to identify the six spatial types

\section{Case 1: Yuyuan Garden}

The Yuyuan Garden is located in the city centre of Shanghai and has an area of approximately $20,000 \mathrm{~m}^{2}$. Parts of the garden were destroyed during the Second World War and have since been repaired or rebuilt. The Yuyuan garden is well known for its delicate and subtle planning and for its artificial mountain and the water feature in its centre. Table 1 shows the connectivity analysis results for the Yuyuan garden graph. The pavilion has the highest $M D$ value $(M D=4.73)$, which means making it the most isolated spatial type, and the covered corridor has the lowest $M D$ value $(M D=3.25)$. The integration value suggests that the pathway is 
Fig. 4 Yuyuan Garden plan, depthmap analysis
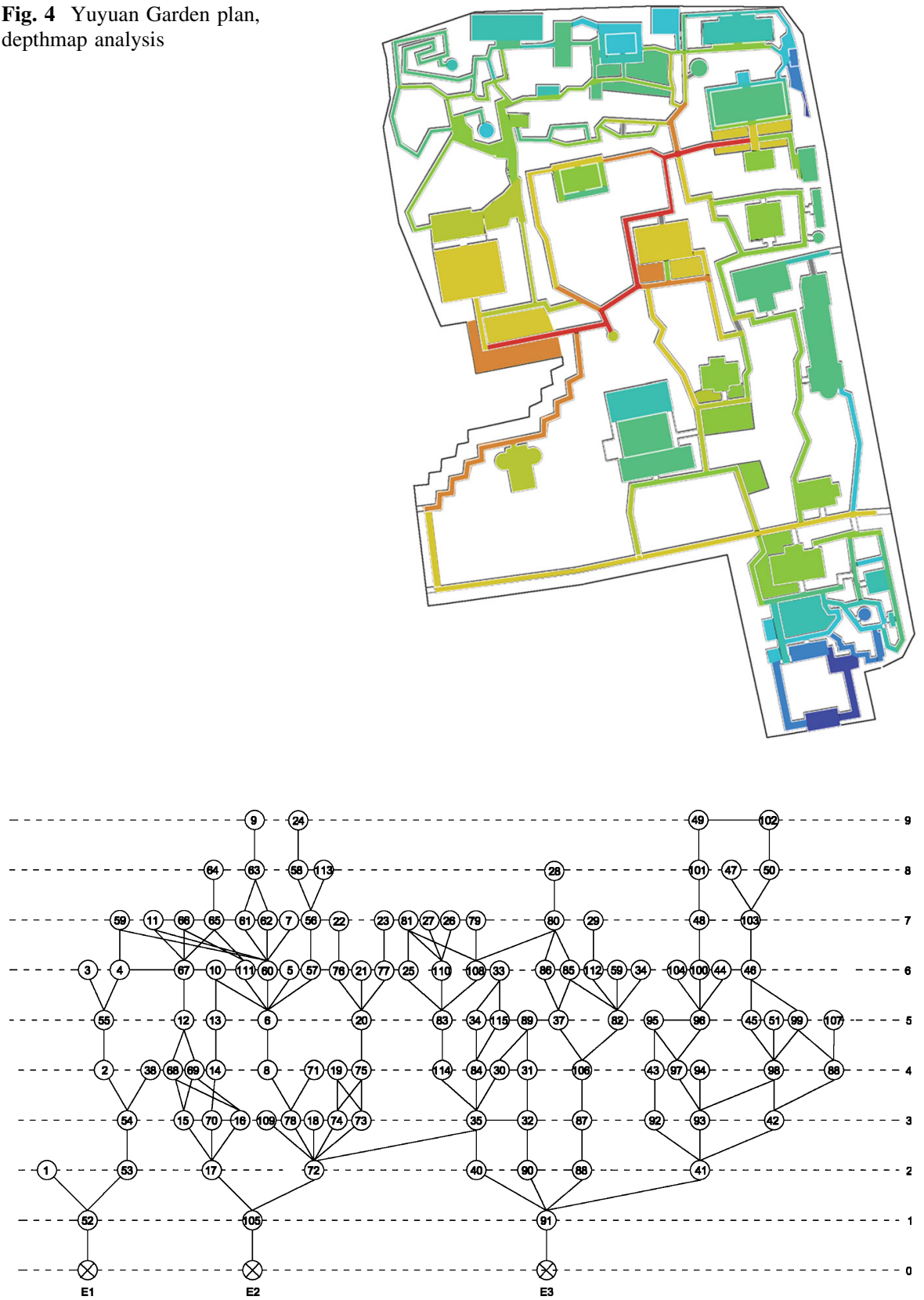

Fig. 5 Yuyuan Garden, connectivity graph representation of the data

the most integrated space $(i=12.04)$, and the least is the covered corridor $(i=0.27)$. The results confirm the common expectation because in TCPGs the pathway generally provides the major connection to other spatial types. 
Table 1 Connectivity analysis of Yuyuan Garden (mean results for $T D, M D$ and $i$ for each spatial type)

\begin{tabular}{llcccr}
\hline Spatial type & Number of spaces & $T D$ & $M D$ & \multicolumn{1}{l}{$S D$ of $M D$} & $i$ \\
\hline Large room & 10 & 39 & 4.33 & 1.29 & 1.20 \\
Small room & 18 & 79 & 4.65 & 2.28 & 2.19 \\
Pavilion & 12 & 52 & 4.73 & 1.56 & 1.34 \\
Yard/square & 7 & 22 & 3.67 & 2.54 & 0.94 \\
Covered corridor & 5 & 13 & 3.25 & 1.67 & 0.67 \\
Pathway & 63 & 219 & 3.53 & 2.31 & 12.04 \\
\hline
\end{tabular}

Table 2 Connectivity analysis of Zhuozhengyuan Garden (mean results for $T D, M D$ and $i$ for each spatial type)

\begin{tabular}{lccccr}
\hline Spatial type & Number of spaces & $T D$ & $M D$ & $S D$ of $M D$ & $i$ \\
\hline Large room & 9 & 42 & 5.25 & 2.45 & 0.82 \\
Small room & 14 & 64 & 4.92 & 2.06 & 1.53 \\
Pavilion & 13 & 74 & 6.17 & 1.93 & 1.06 \\
Yard/square & 11 & 61 & 6.10 & 2.02 & 0.88 \\
Covered corridor & 3 & 11 & 5.50 & 1.15 & 0.11 \\
Pathway & 62 & 243 & 3.98 & 2.38 & 10.05 \\
\hline
\end{tabular}

Table 3 Connectivity analysis of Liuyuan Garden (mean results for $T D, M D$ and $i$ for each spatial type)

\begin{tabular}{lrrrrr}
\hline Spatial type & Number of spaces & $T D$ & $M D$ & $S D$ of $M D$ & $i$ \\
\hline Large room & 7 & 36 & 6.00 & 1.35 & 0.50 \\
Small room & 13 & 81 & 6.75 & 2.28 & 0.96 \\
Pavilion & 6 & 34 & 6.80 & 2.34 & 0.34 \\
Yard/square & 10 & 52 & 5.78 & 2.66 & 0.84 \\
Covered corridor & 6 & 42 & 8.40 & 0.89 & 0.27 \\
Pathway & 33 & 186 & 5.81 & 2.38 & 3.22 \\
\hline
\end{tabular}

\section{Case 2: Zhuozhengyuan Garden}

Zhuozhengyuan Garden is located in Suzhou and it has an area of around $41,334 \mathrm{~m}^{2}$. There are three main parts to the garden, although its essence is regarded as being found in the middle section. Water in the Zhuozhengyuan garden occupies almost one-third of its area, with its pavilions, artificial mountains and other structures all following the water's edge. As shown in Table 2, the pavilion spatial type has the highest mean depth $(M D=6.17)$. This is followed by the courtyard $(M D=6.10)$ and the path type has the lowest $(M D=3.98)$; which is the same pattern as the one found in the Yuyuan garden. The integration value (i) also shows that the pathway is the most integrated space $(i=10.05)$ and the least is the covered corridor $(i=0.11)$. 


\section{Case 3: Liuyuan Garden}

Liuyuan garden is also located in Suzhou and it occupies approximately $23,300 \mathrm{~m}^{2}$. There are four main parts in this garden, the middle section being dominated by water and an artificial mountain. The highest mean depth $(M D=8.40)$ is for the covered corridor, followed by the pavilion $(M D=6.80)$ (Table 3). This differs from the other TCPGs tested, because in the Liuyuan garden the covered corridor is the most isolated space. However, as with the other two, the integration values (i) suggest that the pathway is the most integrated space $(i=3.22)$ and the least integrated is the covered corridor $(i=0.27)$.

In summary, the results derived from the three historic TCPGs suggest the following. First, for all of the gardens the most integrated spatial type is the pathway, followed by the small garden room and the least integrated is the covered corridor. Second, for the Yuyuan and the Zhuozhengyuan gardens, the pavilion type has the highest mean depth, and for Liuyuan garden, the highest mean depth is the covered corridor, followed by the pavilion. Finally, two of the gardens (Zhuozhengyuan and Liuyuan) have the smallest mean depth value for the pathway. For the Yuyuan garden the smallest mean depth value is covered corridor. These results confirm that for the different spatial types in these three TCPGs, there are some common mathematical characteristics that can be generalised. Furthermore, the connectivity analysis based on $M D$ and $i$ values can be meaningfully used to explore the spatial characteristics of TCPGs.

\section{TCPG Inequality Genotype}

An "inequality genotype" is a ranking of spaces, or in the present case spatial types, in order from the highest to the lowest $i$ values (Bafna 2001). Table 4 shows the average connectivity analysis values of the three TCPG cases. Thus, in this case the spatial types are ranked according to the average $i$ value for each type, from the highest to the lowest, as follows: pathway $>$ small garden room $>$ pavilion $>$ yard $>$ large garden room $>$ covered corridor. However, the value differences between three of the spatial types-the pavilion $(i=0.92)$, yard $(i=0.89)$ and large garden room $(i=0.84)$ —are relatively minor. Therefore, for the purposes of this stage they have been ranked jointly to produce a simplified inequality genotype: pathway $>$ small garden room $>$ pavilion/yard/large garden room $>$ covered

Table 4 Average values of the convex space analysis of the three garden cases

\begin{tabular}{lcccr}
\hline Spatial type & Number of spaces & $T D$ & $M D$ & $i$ \\
\hline Large room & 8.67 & 39.00 & 5.19 & 0.84 \\
Small room & 15.00 & 74.67 & 5.44 & 1.56 \\
Pavilion & 10.33 & 53.33 & 5.90 & 0.92 \\
Yard/square & 9.33 & 45.00 & 5.18 & 0.89 \\
Covered corridor & 4.67 & 22.00 & 5.72 & 0.35 \\
Pathway & 52.67 & 216.00 & 4.44 & 8.44 \\
\hline
\end{tabular}


Table 5 Testing range of $i$ values

\begin{tabular}{llll}
\hline Spatial type & $i$ & $S D$ of $i$ & Testing range $(i$-range $)$ \\
\hline Large room & 0.84 & 0.35 & $0.49-1.19$ \\
Small room & 1.56 & 0.62 & $0.96-2.18$ \\
Pavilion & 0.92 & 0.51 & $0.40-1.43$ \\
Yard/square & 0.89 & 0.05 & $0.84-0.94$ \\
Covered corridor & 0.35 & 0.29 & $0.06-0.64$ \\
Pathway & 8.44 & 4.63 & $3.81-13.07$ \\
\hline
\end{tabular}

corridor. Furthermore, in order to determine a more precise set of limits for the following stage of the research, the standard deviation $(S D)$ of the $i$ value was calculated to set its testing range (Table 5). According to the testing range, the Yuyuan garden has three spatial types within the range (pavilion, yard/square, path way) and the Liuyuan garden has four (large room, small room, yard/square, covered corridor) while all of the space types in the Zhuozhengyuan garden are within the $i$-range tested.

\section{Stage 2: Parametric Generation}

A parameter is a value or measurement that can be altered or changed. Parametric systems focus on the representation and control of the relationships between objects, supporting the creation, management and organisation of complex design models (Woodbury 2007). Using parametric tools, designers can make rules that reflect the performance requirements of a design and then generate variants that fulfil these conditions. By changing parameters, particular instances can be created from a potentially infinite range of possibilities (Kolarevic 2003). Thus, this approach provides a description of a design using parameters and relationships that allow for variations to be developed and tested to produce compliant or optimal design solutions (Monedero 2000; Gane and Haymaker 2009). In the form-finding process, the use of parametric methods can enhance design flexibility and control (Fischer 2003). To a large extent, the geometry modelling approach in parametric design is dependent on variation settings (Burry 2003; Baerlecken 2010). Parametric design software includes Generative Component, Digital Project and Grasshopper. Scripting tools include Processing (based on the Java language), Rhino script and Python script (based on VB language) and DesignScript. In the present study, Grasshopper was used for the scripting and generation of the new TCPGs. Significantly, despite the large volume of research published about generating novel forms using parametric scripts, their use to replicate existing or historic conditions remains uncommon.

The basic parameters used to generate the new TCPGs for the present paper include the number, type and mean connectivity relations (expressed using $T D, M D$ and $i$ ) in the three historic cases. Several researchers have previously proposed using 
such syntactical measures as parameters. For example, Jeong and Ban (2011) developed computational algorithms to evaluate design solutions using space syntax methods. Nourian et al. (2013) presented a computational toolkit developed for configurative architectural design using space syntax theory. Their toolkit, named "SYNTACTIC", is a plugin for Grasshopper and is useful for generating spatial diagrams and providing real-time evaluation.

Using the mathematical characteristics of the three historic TCPGs as a basis, a parametric system was authored to generate a series of spatial type and connectivity graphs which conform to these characteristics. Specifically, for each of the three historic TCPG sites, three new mathematically compliant garden plans were generated (nine on total demonstrated here). The efficacy of the generative system was determined in each of the cases by checking $T D, M D$ and $i$ against the inequality genotype, identified in the case studies, and $S D$ measures for each value. The average size and number of each spatial type was used to generate nodes, then connectivity points were optimised to achieve the graph values, and finally the nodes transformed into abstract, but correctly scaled, garden elements. At the end of the process, each of the nine new gardens were made up of distinct spatial types and the connections between them. The following subsections describe how the connectivity rules were produced and the means by which the nodes were resized to replicate the spatial types.

\section{Steps in the generative and testing process}

In order to generate a parametric system which reflects the mathematical characteristics of the TCPGs, the following steps were taken.

- First, some mathematical characteristics of different spatial types derived from the three TCPG cases were set as rules in Grasshopper. For instance, the pavilion typically has only one connection; a proportion of the yards are typically connected to large garden rooms, etc.

- Second, the average number of each spatial type in the TCPG-such as the number of large rooms or small rooms in a garden-was then set as input parameters.

- Third, for this demonstration a pre-determined entrance, generic site boundary and main path curve were selected, as well as the distance range of the nodes to the primary path.

- Fourth, new parametrically generated plans of TCPGs were produced, each suggesting a possible connectivity planning schema reflecting the characteristics of the historic TCPGs.

- Finally, the $T D, M D, i$ values of the generated parametric diagram were tested against the inequality genotypes produced for the three TCPG cases.

When performing the $i$-range testing of an example parametric plan, if the $i$ value was within the range (see Table 5), the outcome was classed as "true". The more "true" values the system generates, the more closely the characteristics of the new TCPG design reflects those of the historic TCPGs. As the historic cases all have at least three spatial types that are within the $i$-test range, in order to be confirmed as a 
"compliant" landscape design, a filtering criteria was set which confirmed that the new parametric diagram must have at least three "true" values.

Figure 6 and Table 6 show an example of a parametrically generated and tested connectivity diagram of a new TCPG produced in accordance with these four steps. In this figure, different nodal symbols represent the six spatial types in the garden along with a seventh for the entrance. The testing of the parametric diagram in the example confirms that it complies with the inequality genotype test and, in terms of the $i$-range test, four of the six properties are "true", meaning that the diagram exceeds the minimum characteristic of the TCPG.

\section{Forming and sizing the spatial types}

In order to complete the garden plan derived from the parametrically generated connectivity diagram, a method was required to convert the spatial-type nodes into

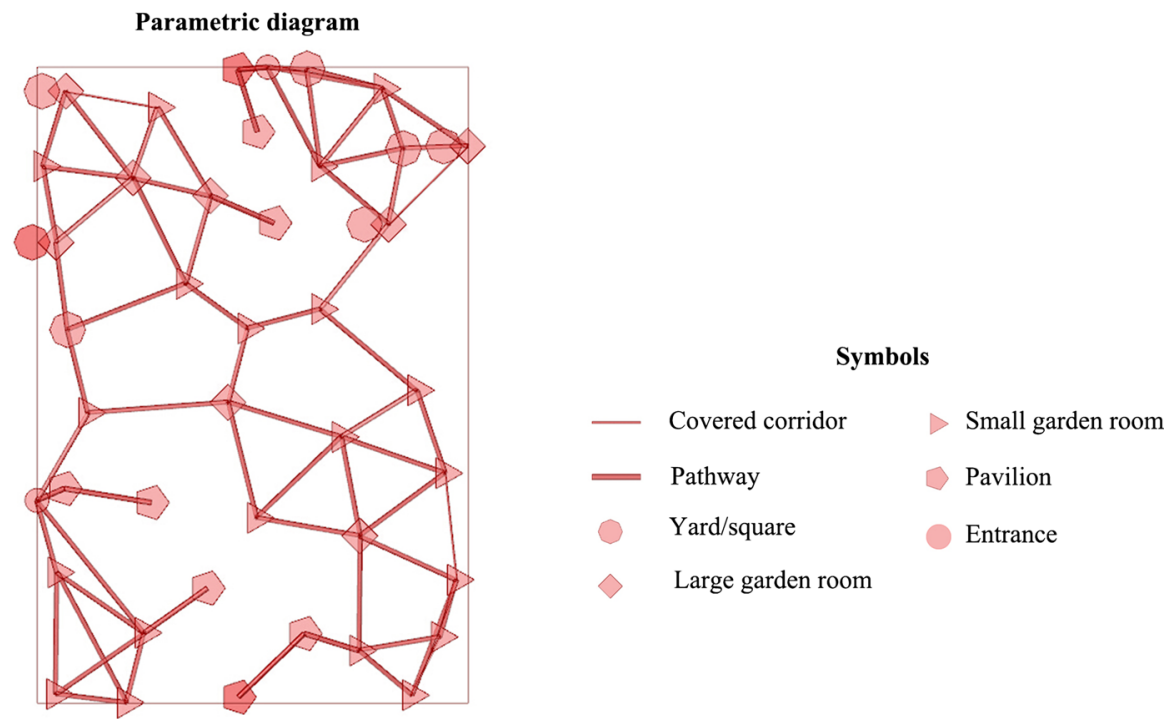

Fig. 6 An example parametric diagram generated using TCPG connectivity measures

Table 6 Compliance testing for the example parametric diagram

\begin{tabular}{lcccccc}
\hline Spatial type & $\begin{array}{l}\text { Number } \\
\text { of spaces }\end{array}$ & $T D$ & $M D$ & $i$ & $\begin{array}{l}\text { Inequality } \\
\text { genotype rank }\end{array}$ & $i$-range test \\
\hline Large room & 20 & 24 & 3.43 & 1.24 & 3 & False \\
Small room & 8 & 64 & 3.37 & 3.80 & 2 & False \\
Pavilion & 8 & 24 & 3.43 & 1.24 & 3 & True \\
Yard/square & 7 & 23 & 3.83 & 0.88 & 5 & True \\
Covered corridor & 4 & 12 & 4.00 & 0.33 & 6 & True \\
Pathway & 55 & 177 & 3.28 & 11.63 & 1 & True \\
\hline
\end{tabular}


correctly scaled, geometric forms, and the paths connecting them into practical, topographically correct routes. The first of these was achieved by comparing the size of each of the six spatial types found in the three historic cases. In this way a range could be determined for each spatial type. Table 7 records the size range and common form or shape of four of the garden elements in the historic cases.

The covered corridor and pathway shapes and locations in the garden plans present a different challenge. Their typical widths were determined using those in the historic cases, and their directions were prioritized to run parallel to the dominant site boundary as part of their connecting role. This is because, in the historic cases, there are two types of path form: one is parallel with the site boundary and the other is more organic. In this study, a rule was produced to

Table 7 Form and size for each functional type (all units are in square meters)

\begin{tabular}{lllll}
\hline & Large garden room & Small garden room & Pavilion & Square \\
\hline Plan shape & Rectangle & Rectangle & Octagonal & Rectangle \\
Mean & 148.47 & 50.17 & 19.55 & 99.42 \\
SD & 71.67 & 31.80 & 17.50 & 52.37 \\
Size range & $75.80-220.14$ & $18.37-81.97$ & $2.05-37.05$ & $47.05-151.79$ \\
\hline
\end{tabular}

Fig. 7 An example parametric plan generated in accordance with connectivity value rules and modified in response to spatial size and shape rules

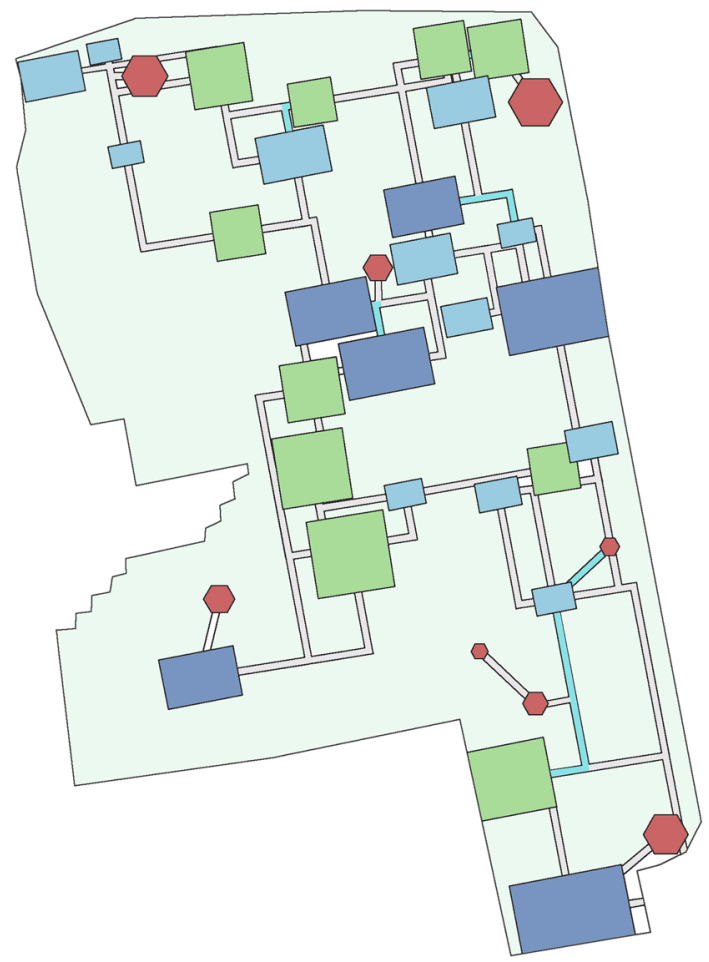


replicate the parallel pathway system only, the organic pathways being beyond the scope of the present research.

With these two sets of rules implemented, the new connectivity graph was converted into a garden plan layout, with scaled and appropriately shaped elements and connections. Figure 7 is an example of a newly generated garden plan using the Yuyuan Garden site boundary.

\section{Parametrically generated examples}

To complete this stage, for each of the three historic sites, three new compliant plans were produced (Tables 8, 9, 10). Compliant in this instance means corresponding to the inequality genotype and having at least three "true" determinants in the range measures. Thus, the new plans closely replicate the connectivity characteristics of the historic TCPGs. More specifically, from the $i$-range testing of newly generated plans it can be seen that all of the pavilions are within the tested range, followed by the large garden room and the covered corridor. The functional type with the lowest level of compliance is the small garden room followed by the Yard/Square. This suggests that the functional type of pavilions complies with the spatial connectivity characteristics of TCPG best, while small garden rooms and yard/square types are less compliant.

\section{Stage 3: Fractal Analysis}

A fractal dimension is a non-integer value that represents the characteristic or typical distribution of detail or form in an image or object (Mandelbrot 1977, 1982). A fractal dimension could therefore be thought of as a statistical measure of visual complexity or textural roughness. For a two-dimensional image, the fractal dimension $(D)$ is a value between 1.0 and 2.0, where a higher value of $D$, closer to 2.0, suggests a visually complex image. Fractal dimensions have repeatedly been used since the early 1990s to analyse and compare the visual character of urban space (Batty and Longley 1994; Stamps 2002; Chalup et al. 2009) and architecture (Bovill 1996; Lorenz 2003; Ostwald et al. 2015). Significantly, fractal dimensions have also been used to analyse the Yuyuan garden ( $\mathrm{Lu} \mathrm{2010)}$ and the visual complexity of urban squares and gardens in China (Liang et al. 2013).

The standard mathematical method for calculating the fractal dimension of a twodimensional image has been presented previously on multiple occasions (Mandelbrot 1982; Bovill 1996; Lorenz 2003). This approach, called the box-counting method, has also been substantially refined in recent years and the optimal method of application and settings for the process determined (Ostwald 2013; Ostwald and Vaughan 2013a, b). These methodological settings and standards are used for the present research.

The three historic and nine new TCPG plans were each analysed using ArchImage software, and their fractal dimensions calculated (Table 11). For each of the new plans, the difference between its $D$ value, and the $D$ value of the original 
Table 8 Comparison of the newly generated Yuyuan Garden plans with the historical plan (spatial types are coded as per the key in Fig. 3)

\begin{tabular}{|c|c|c|c|}
\hline \multirow{7}{*}{ Original plan } & Functional type & $i$ & $i$ test \\
\hline & Large garden room & 1.20 & True \\
\hline & Small garden room & 2.19 & True \\
\hline & Pavilion & 1.34 & True \\
\hline & Yard/Square & 0.94 & True \\
\hline & Covered corridor & 0.67 & False \\
\hline & Path way & 12.04 & True \\
\hline \multirow{7}{*}{ New plan 1} & Functional type & $\bar{i}$ & $i$ test \\
\hline & Large garden room & 0.94 & True \\
\hline & Small garden room & 2.04 & True \\
\hline & Pavilion & 1.31 & True \\
\hline & Yard/Square & 1.80 & False \\
\hline & Covered corridor & 0.83 & False \\
\hline & Path way & 10.54 & True \\
\hline \multirow[t]{7}{*}{ New plan 2} & Functional type & $i$ & $i$ test \\
\hline & Large garden room & 1.07 & True \\
\hline & Small garden room & 3.83 & False \\
\hline & Pavilion & 0.83 & True \\
\hline & Yard/Square & 0.83 & False \\
\hline & Covered corridor & 0.60 & True \\
\hline & Path way & 12.1 & True \\
\hline \multirow[t]{7}{*}{ New plan 3} & Functional type & $i$ & $i$ test \\
\hline & Large garden room & 0.75 & True \\
\hline & Small garden room & 3.00 & False \\
\hline & Pavilion & 1.25 & True \\
\hline & Yard/Square & 2.33 & False \\
\hline & Covered corridor & 0.43 & True \\
\hline & Path way & 11.27 & True \\
\hline
\end{tabular}

was calculated and presented as a percentage (\% Diff), along with a positive or negative indicator of whether it is higher or lower (more or less visually complex) than the corresponding original. As shown in Table 11, there are two ways to compare these mean values. The "relative" variation includes the positive and negative values in its mean calculation. The "absolute" variation treats all differences as positives regardless of whether they are higher or lower than the 
Table 9 Comparison of the newly generated Zhuozhengyuan Garden plans with the historical plan

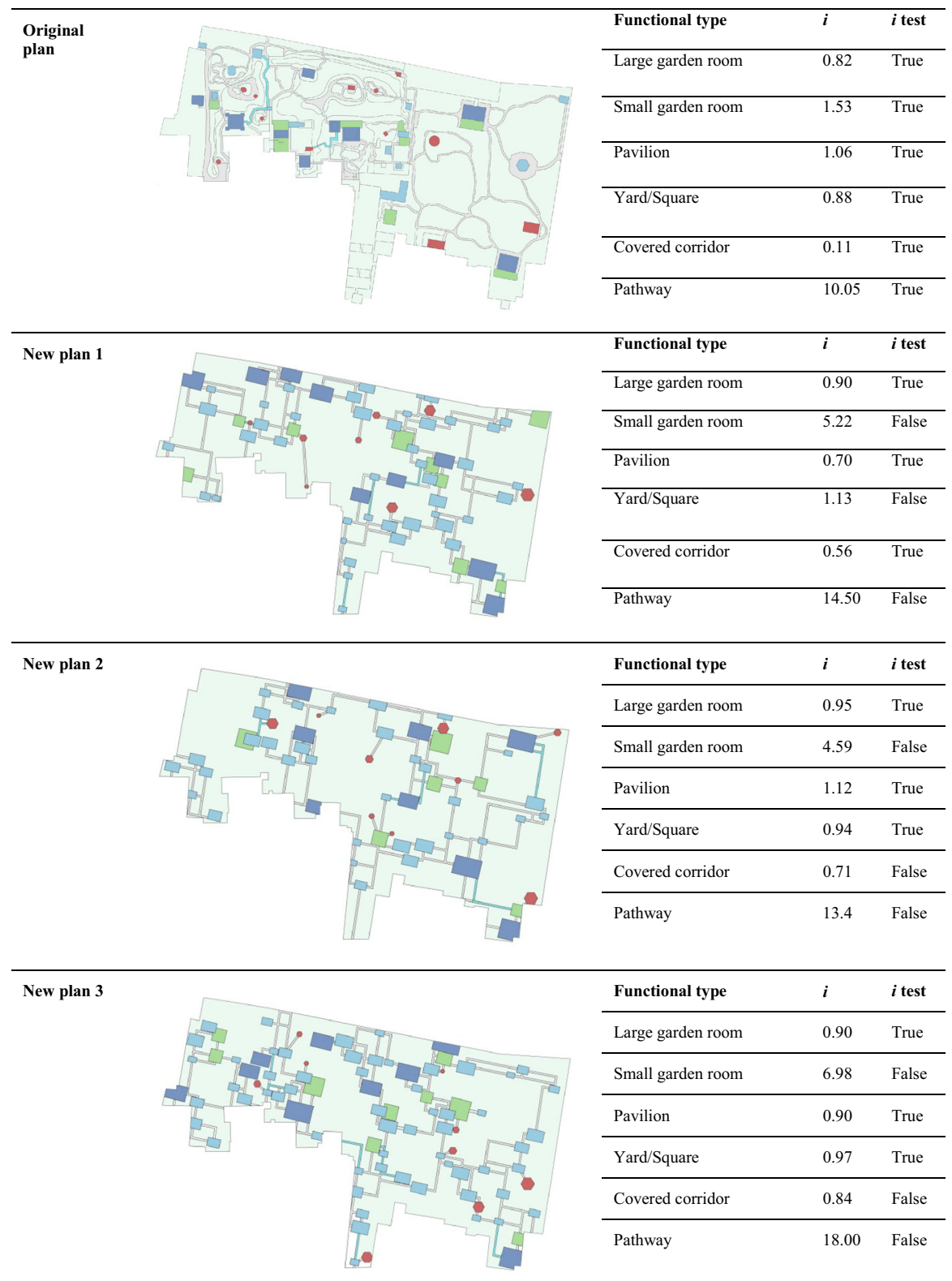

target. The former is the more conventional method in fractal analysis as it takes into account the fact that a trend in higher or lower results is potentially important to accommodate into the mean. However, for measuring the raw similarities between a target figure and variations, the latter, absolute value may be more useful. 
Table 10 Comparison of the newly generated Liuyuan Garden plans with historical plan

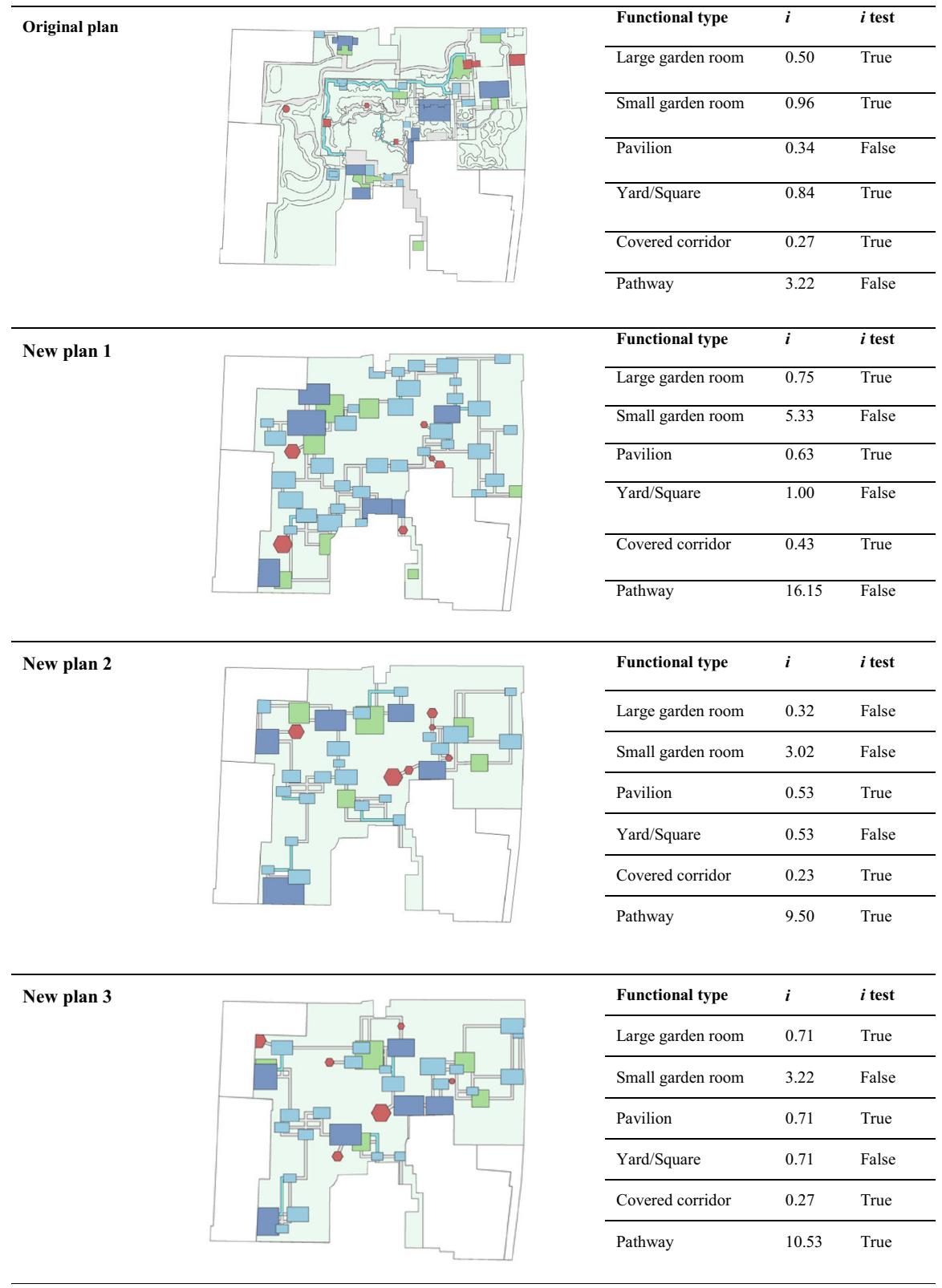

For the relative mean, the result for the Yuyuan gardens site was $2.6 \%$ less visually complex than the original and for the Zhuozhengyuan and Liuyuan sites, $7 \%$ less and $8.3 \%$ less, respectively. Interpretation of these numerical results can be undertaken in two ways. First, it is possible to describe a difference of less than 
Table 11 Fractal dimensions $(D)$ for the three original TCPGs and three new versions for each site and their relative difference from the original

\begin{tabular}{|c|c|c|c|c|c|c|c|c|c|}
\hline \multirow[t]{2}{*}{ Site } & \multirow{2}{*}{$\begin{array}{l}\text { Original } \\
D\end{array}$} & \multicolumn{2}{|c|}{$\begin{array}{l}\text { New } \\
\text { Version } 1\end{array}$} & \multicolumn{2}{|c|}{$\begin{array}{l}\text { New } \\
\text { Version } 2\end{array}$} & \multicolumn{2}{|c|}{$\begin{array}{l}\text { New } \\
\text { Version } 3\end{array}$} & \multicolumn{2}{|l|}{ Summary } \\
\hline & & $D$ & $\%$ Diff & $D$ & $\%$ Diff & $D$ & $\%$ Diff & $\begin{array}{l}\text { Mean \% } \\
\text { Diff } \\
\text { relative }\end{array}$ & $\begin{array}{l}\text { Mean \% } \\
\text { Diff } \\
\text { absolute }\end{array}$ \\
\hline Yиуиап & 1.58 & 1.46 & -12 & 1.46 & -12 & 1.74 & +16 & -2.6 & 13.3 \\
\hline Zhuozhengyuan & 1.55 & 1.48 & -7 & 1.46 & -9 & 1.50 & -5 & -7 & 7 \\
\hline Liuyuan & 1.52 & 1.42 & -10 & 1.43 & -9 & 1.46 & -6 & -8.3 & 8.3 \\
\hline
\end{tabular}

$5 \%$ as suggesting a "very similar" level of appearance, while between 5 and $10 \%$ implies a shift from "similar" to "comparable" and above $10 \%$, dissimilar. In this way, the absolute result for the Yuyuan gardens is very similar, and the other two only slightly less so. Secondly, all three means are below the target. That is, they are consistently less complex than the original.

For the absolute mean difference, the Yuyuan gardens site result was $13.3 \%$, the Liuyuan $8.3 \%$ and the Zhuozhengyuan $7 \%$. In absolute terms, all three range from being visually comparable to becoming dissimilar. Furthermore, the worst absolute result, for the Yuyuan gardens, was the best of the relative results. This is because the absolute results do not provide any indication of higher or lower dimensionality. Thus, the relative result is the most useful of the two for interpreting the difference, while the absolute is a better indicator of which set of parametrically produced plans is closest to its original.

For the final comparison, the new versions are classified in accordance with percentage difference and $i$-test results (Table 12). From these results it is apparent that newly generated plans for the Liuyuan and Zhuozhengyuan sites have very similar or close levels of relative complexity in comparison with the original plans. However, all of the newly generated plans on the Yuyuan Garden site are more divergent from the results of the original plans in terms of visual complexity. No clear explanation for this trend was detected in the results, but it is notable that of the three historical sites, the Yuyuan garden has the smallest extent of curvilinear paths, something that was not explicitly modelled in the parametric script. A comparison of $i$-test (true/false compliant) results with fractal dimension measures demonstrates that the Yuyuan garden site produced more compliant results (four out of six true) than the other two sets of gardens, and yet it had the least similar levels of visual complexity. This is a seemingly paradoxical outcome, which prima facie suggests that the more rigidly compliant the socio-spatial properties of the plan are, the less aesthetically related to its original it is. However, the set of results is too small to examine this issue further, and the differences identified are not necessarily substantial enough to support such a conclusion $(<10 \%$ in $D$ and $15 \%$ in $i$-test). Conversely, of the complete set of compliant plans, the majority were "very similar", or "similar" in appearance, solely on the basis of sociospatial structure. 
Table 12 Grouping of newly generated plans by fractal dimension differences, compared with compliance levels

\begin{tabular}{|c|c|c|c|c|c|}
\hline & $\begin{array}{l}\text { Threshold } \\
(\%)\end{array}$ & Description & Generated plans & $\%$ Diff & $\begin{array}{l}i \text {-test true/false } \\
\text { (original) }\end{array}$ \\
\hline Group 1 & $2-5$ & $\begin{array}{l}\text { Very similar } \\
\text { or alike }\end{array}$ & $\begin{array}{r}\text { New version } 3 \text { of } \\
\text { Zhuozhengyuan }\end{array}$ & -5 & $3 / 3(6 / 6)$ \\
\hline \multirow[t]{5}{*}{ Group 2} & \multirow[t]{5}{*}{$6-10$} & \multirow[t]{5}{*}{$\begin{array}{l}\text { Similar or } \\
\text { comparable }\end{array}$} & $\begin{array}{l}\text { New version } 2 \text { of } \\
\text { Zhuozhengyuan }\end{array}$ & -9 & $3 / 3(6 / 6)$ \\
\hline & & & $\begin{array}{l}\text { New version } 1 \text { of } \\
\text { Zhuozhengyuan }\end{array}$ & -7 & $3 / 3(6 / 6)$ \\
\hline & & & New version 1 of Liuyuan & -10 & $3 / 3(4 / 2)$ \\
\hline & & & New version 2 of Liuyuan & -9 & $3 / 3(4 / 2)$ \\
\hline & & & New version 3 of Liuyuan & -6 & $3 / 3(4 / 2)$ \\
\hline \multirow[t]{3}{*}{ Group 3} & \multirow[t]{3}{*}{$>10$} & \multirow[t]{3}{*}{ Dissimilar } & New version 1 of Yuyuan & -12 & $4 / 2(5 / 1)$ \\
\hline & & & New version 2 of Yuyuan & -12 & $4 / 2(5 / 1)$ \\
\hline & & & New version 3 of Yuyuan & +16 & $4 / 2(5 / 1)$ \\
\hline
\end{tabular}

\section{Conclusion}

This paper demonstrates a method for generating new garden designs that conform to selected socio-spatial patterns present in sixteenth-century TCPGs and, by doing so, producing plans which have broadly similar levels of visual richness and complexity. Through this research an important new application of generative design is proposed, along with a potential new insight into the visual and geometric character of TCPGs.

For the first of these outcomes, the research has presented a parametric system for generating new variations of an existing complex spatial structure using a limited set of syntactical values as rules. This is a flexible system that is appropriate for producing new plan variants at the conceptual design stage. When multiple designs are generated, based on the parametric rule sets, the plans comply with the character of its original site or context, leading to potential applications for landscape designers.

The second outcome of the research is the observation that the parametric system demonstrated in this paper was able to generate garden plans which are visually similar or comparable to the original TCPGs using only connectivity measures. This implies that a large part of the much-celebrated visual character of the TCPG may be derived from the structure of its network of spaces. This might be a reasonable assumption given that, for example, in the Yuyuan garden there are 115 connected areas conforming to six major spatial types, all of which are constrained within a complex site boundary. Thus, the visual complexity of the plan may well be directly shaped by its socio-spatial structure.

Finally, by extending this research to consider a larger number of historic cases, or those from different regions or eras, the mathematical characteristics (sociospatial and fractal dimensions) of these TCPGs may be compared and differentiated to reveal new insights into these historic, and culturally important, environments. 


\section{References}

Baerlecken, D., M. Martin, R. Judith and K. Arne. 2010. Integrative parametric form-finding processes. In: New Frontiers, 15th CAADRIA Conference Proceedings, 303-312.

Bafna, S. 2001. Geometric intuitions of genotypes. In: Proceedings of the 3rd international symposium on space syntax (Atlanta, July 2001), ed. J. Peponis, J. Wineman, \& S. Bafna, 20.21-20.16. Michegan: Alfred Taubman College of Architecture and Urban Planning, University of Michigan.

Bafna, S. 2003. Space syntax: a brief introduction to its logic and analytical techniques. Environment and Behavior 35 (1): 17-29.

Batty, M. and P. Longley. 1994. Fractal Cities: A Geometry of Form and Function. San Diego: Academic Press.

Bovill, C. 1996. Fractal Geometry in Architecture and Design. Swizerland: Birkhäuser.

Burry, M. 2003. Between intuition and process: parametric design and rapid prototyping. In: Architecture in the Digital Age: Design and Manufacturing, ed. B. Kolarevic, 149-162. New York and London: Spon Press.

Chalup, S. K., N. Henderson, M. J. Ostwald and L. Wiklendt. 2009. A Computational Approach to Fractal Analysis of a Cityscape's Skyline. Architectural Science Review 52 (2): 126-134.

Chang, H. Y. 2006. The Spatial Structure Form of Traditional Chinese Garden-A Case Study on The Lin Family Garden. Master thesis, National Chenggong University.

Chen, G. 2011. Planting Design Illustrated. Irvine: ArchiteG.

Chen, R. 2012. Research on Traditional Chinese Garden's Space Syntax and Insights into Contemporary Regional Reconstruction. Master Thesis, Tsinghua University.

Feng, X. 2010. The Great Gardens of China: History, Concepts, Techniques. New York: The Monacelli Press.

Fischer, M., M. Burry and J. Frazer. 2003. Triangulation of Generative Form for Parametric Design and Rapid Prototyping. In: Digital design, 21st eCAADe Conference Proceedings, 441-448.

Fu, X., D. Guo, X. Liu, G. Pan, Y. Qiao, D. Sun and N. S. Steinhardt. 2002. Chinese Architecture. New Haven: Yale University Press.

Gane, V. and J. Haymaker. 2009. Design scenarios: Methodology for requirements driven parametric modeling of high-rises. In: Proceedings of the 9th International Conference on Construction Applications of Virtual Reality (CONVR 2009, Sydney, Nov 2009), eds. N. Gu and X. Wang, 79-90. Sydney: University of Sydney.

Guo, J. 2014. Application of Depth Map Software in Spatial Structure Analysis of Master-of-nets Garden. Chinese Landscape Architecture 8, 120-124.

Hanson, J. 1998. Decoding Homes and Houses. Cambridge: Cambridge University Press.

Hillier, B. 1995. Space is the Machine. Cambridge: Cambridge University Press.

Hillier, B. and J. Hanson. 1984. The Social Logic of Space. Cambridge: Cambridge University Press.

Hillier, B. and T. Kali. 2006. Space syntax: the language of museum space. In: A Companion to Museum Studies, ed. M. Sharon, 282-301. London: Blackwell.

Hunt, J. D. 2012. A World of Gardens. London: Reaktion.

Jeong, S. K. and Y. U. Ban. 2011. Computational algorithms to evaluate design solutions using Space Syntax. Computer-Aided Design 43 (6): 664-676.

Keswick, M. 1978. The Chinese Garden: History, Art and Architecture. New York: St. Martin's Press.

Keswick, M., C. Jencks. and A. Hardie. 2003. The Chinese Garden: History, Art and Architecture. Massachusetts: Harvard University Press.

Kolarevic, B. 2003. Architecture in the Digital Age : Design and Manufacturing. New York: Spon Press.

Li, Z. 2011. Visual perception of traditional garden space in Suzhou, China: A case study with space syntax techniques. In: Proceedings of 19th International Conference on Geoinformatics (Shanghai, June 2011), ed. X. Li and S. Bao, 1-4. Middlesex: Piscataway, N.J.

Liang, J., Y. Hu and H. Sun. 2013. The Design Evaluation of the Green Space Layout of Urban Squares Based on Fractal Theory. Nexus Network Journal 15 (1): 33-49.

Lorenz, W. E. 2003. Fractals and fractal architecture. Master thesis, Vienna University of Technology.

Lu, S. 2009. From Syntax to Plot: the spatial language of a Chinese garden. In: Proceedings of the 7th International Space Syntax Symposium (Stockholm, June 2009), ed. D. Koch, L.Marcus and J.Steen, 067:1-067:15. Stockholm: KTH.

Lu, S. 2010. Hidden orders in Chinese gardens: irregular fractal structure and its generative rules. Environment and Planning B: Planning and Design 37 (6): 1076-1094. 
Mandelbrot, B. B. 1977. Fractals: Form, Chance, and Dimension. New York: W. H. Freeman \& Company.

Mandelbrot, B. B. 1982. The Fractal Geometry of Nature. New York: W. H. Freeman and Company.

Minor, E. S. and D. Urban. 2007. Graph theory as a proxy for spatially explicit population models in conservation planning. Ecological Applications 17: 1771-1782.

Monedero, J. 2000. Parametric design: a review and some experiences. Automation in Construction 9 (4): 369-377.

Nourian, P., S. Rezvani and S. Sariyildiz. 2013. A syntactic architectural design methodology: Integrating real-time space syntax analysis in a configurative architectural design process. In: Proceedings of the 9th International Space Syntax Symposium (Seoul, November 2013), ed. Y. O. Kim, H. T. Park, K. W. Seo, 048: 041-015. Seoul: Sejong University Press.

Ostwald, M. J. 2011. The mathematics of spatial configuration: Revisiting, revising and critiquing justified plan graph theory. Nexus Network Journal 13 (2): 445-470.

Ostwald, M. J. 2013. The Fractal Analysis of Architecture: Calibrating the Box-Counting Method Using Scaling Coefficient and Grid Disposition Variables. Environment and Planning B: Planning and Design 40 (4): 644-663.

Ostwald, M. J. and J. Vaughan. 2013a. Limits and Errors: Optimising Image Pre-Processing Standards for Architectural Fractal Analysis. ArS Architectural Science 7: 1-20.

Ostwald, M. J. and J. Vaughan. 2013b. Representing architecture for fractal analysis: a framework for identifying significant lines. Architectural Science Review 56 (3): 242-251.

Ostwald, M. J., J. Vaughan and C. Tucker. 2015. Characteristic Visual Complexity: Fractal Dimensions in the Architecture of Frank Lloyd Wright and Le Corbusier. In: Architecture and Mathematics from Antiquity to the Future. Vol II. eds. K.Williams and M. J. Ostwald, 339-354. Cham: Springer.

Peng, Y. 1986. Analysis of Chinese Classical Garden. Beijing: China Architecture and Building Press.

Rinaldi, B. M. 2011. The Chinese Garden: Garden Types for Contemporary Landscape Architecture. Basel: Birkhauser.

Stamps, A. E. 2002. Fractals, Skylines, Nature and Beauty. Landscape and Urban Planning 60 (3): $163-184$.

Sui, Y. and W. Xun. 2011. Chinese Gardens. Hong Kong: Design Media Publishing.

Sun, P. 2012. The Contrast Interpretation of Chinese Classical Gardens between Space Syntax Theory and Traditional Theories-The Space Research of Chengde Mountain Resort. Ph.D. thesis, Beijing Forestry University.

Tong, J. 1997. Glimpses of Gardens in Eastern China. Beijing: China Architecture and Building Press. Wang, S. and B. Wang. 2013. Configurational Diagram of Chinese Traditional Architecture: Path Network Analysis in Space Syntax. The Architect 162: 84-90.

Woodbury, R., R. Aish and A. Kilian. 2007. Some Patterns for Parametric Modeling. In: Expanding Bodies: Art-Cities-Environment, 27th ACADIA Conference Proceedings, 222-229.

Zhou, W. 1999. Classical Gardens of China. Beijing: Tsinghua University Press.

Rongrong $\mathbf{Y u}$ is a visiting postdoc fellow at the School of Architecture and Built Environment, the University of Newcastle. Her research interests include computer-aided design, architecture design theory, design cognition. Her $\mathrm{PhD}$ study is focused on exploring designers' behavior in parametric design. A couple of journal and conference papers related to the design cognition and application of parametric design have been published.

Michael J. Ostwald is Professor and Dean of Architecture at the University of Newcastle, Australia. $\mathrm{He}$ is a Visiting Professor at RMIT University and a Research Fellow at SIAL (Melbourne). He has held past academic positions in the USA and Hong Kong and worked for architectural practices in Australia, the UK and the USA. Michael has a PhD in architectural theory and history and a higher doctorate (DSc) in design mathematics. He is Co-Editor-in-Chief of the Nexus Network Journal: Architecture and Mathematics (Springer) and a member of the Editorial Boards of ARQ (Cambridge) and Architectural Theory Review (Taylor \& Francis).

Ning Gu is a widely published scholar in the field of design computing and cognition. His research interests include virtual environments, generative design systems, computational design analysis, 
computer-supported collaborative design, building information modeling (BIM), as well as their applications in the industry and education. His research projects have been supported by major competitive research funding schemes in Australia. He is an associate professor with the School of Architecture and Built Environment at the University of Newcastle, Australia. 